UNIVERSIDADE DE SÃO PAULO

INSTITUTO DE PSICOLOGIA

MÁRCIA CRISTINA MAESSO

Fundamentos do diagnóstico e a posição do analista

SÃO PAULO

2011 


\title{
Fundamentos do diagnóstico e a posição do analista
}

\author{
(versão revisada)
}

Tese apresentada ao Instituto de Psicologia da Universidade de São Paulo, como parte dos requisitos para obtenção do título de Doutor em Psicologia.

Área de concentração: Psicologia Clínica.

Orientadora: Dra. Jussara Falek Brauer

SÃO PAULO

2011 


\section{AUTORIZO A REPRODUÇÃO E DIVULGAÇÃO TOTAL OU PARCIAL DESTE TRABALHO, POR QUALQUER MEIO CONVENCIONAL OU ELETRÔNICO, PARA FINS DE ESTUDO E PESQUISA, DESDE QUE CITADA A FONTE.}

Maesso, Márcia Cristina.

Fundamentos do diagnóstico e a posição do analista / Márcia Cristina Maesso; orientadora Jussara Falek Brauer. -- São Paulo, 2011. $105 \mathrm{f}$.

Tese (Doutorado - Programa de Pós-Graduação em Psicologia. Área de Concentração: Psicologia Clínica) - Instituto de Psicologia da Universidade de São Paulo.

1. Ética. 2. Diagnóstico. 3. Ato. 4. Escrita. 5. Clínica psicanalítica. 6. Posição do analista. I. Título. 
Nome: Maesso, M. C.

Título: Fundamentos do diagnóstico e a posição do analista

$$
\begin{aligned}
& \text { Tese apresentada ao Instituto de Psicologia da } \\
& \text { Universidade de São Paulo para obtenção do título de } \\
& \text { Doutor em Psicologia }
\end{aligned}
$$

Aprovado em:

Banca Examinadora

Profa. Dra. Jussara Falek

Instituição: Instituto de Psicologia - USP Assinatura:

Prof. Dr. Christian Ingo Lenz Dunker

Instituição: Instituto de Psicologia - USP Assinatura:

Prof. Dr. Edson Olivari de Castro

Instituição: UNESP - Bauru

Assinatura:

Profa. Dra. Daniela Scheinkman Chatelard

Instituição: Universidade de Brasília

Assinatura:

Profa. Dra. Rosemeire Aparecida do Nascimento

Instituição: Hospital Universitário - USP Assinatura: 
Ao meu querido companheiro Roberto,

por me dar o que não tem. 


\section{AGRADECIMENTOS}

À minha orientadora doutora Jussara Falek Brauer, pelos anos de convivência e de conversas sempre muito esclarecedoras.

Aos professores doutor Christian Ingo Lenz Dunker e doutor Edson Olivari de Castro, pelas contribuições e sugestões, no momento da qualificação, que foram muito significativas na continuidade da pesquisa.

A Capes pelo apoio financeiro.

A todos que também fizeram parte do LEPPPI e do Projeto Tecer, pois, além do coleguismo, tecemos amizades.

Às parceiras e amigas do Circuito Ponto de Estofo, lugar onde trabalhamos em função da psicanálise.

Às minhas queridas amigas Iara Stabler e Tatiana Assadi, pelos várias formas de apoio.

Aos meus amores Roberto e Tainá.

Aos familiares queridos.

Aos analisandos, pela oportunidade de escutar o inconsciente.

Aos alunos, porque continuamos aprendendo.

Ao menino "Desenhador".

A todos que não mencionei, mas contribuíram de algum modo. 
A gente vive repetido, o repetido, e, escorregável, num mim minuto, já está empurrado noutro galho. Acertasse eu com o que depois sabendo fiquei, para de lá de tantos assombros... Um está sempre no escuro, só no último derradeiro é que clareiam a sala. Digo: o real não está na saída nem na chegada: ele se dispõe para a gente é no meio da travessia.

(João Guimarães Rosa em Grande sertão: veredas) 


\section{RESUMO}

Maesso, M. C. (2011). Fundamentos do diagnóstico e a posição do analista. Tese de Doutorado, Instituto de Psicologia, Universidade de São Paulo, São Paulo.

O trabalho refere-se a uma leitura norteada pela psicanálise e pelas suas proposições éticas, acerca dos fundamentos do diagnóstico, situando inicialmente por meio de Freud, Lacan, e outros autores do campo psicanalítico, o diagnóstico como ato de mestria no discurso médico, para posteriormente formular que o avesso desse discurso constitui a condição de conceber o diagnóstico na psicanálise como semidizer a partir da posição do analista na relação transferencial. Considera a hipótese de que, por meio da escrita do caso clínico, o psicanalista orienta-se pelo Real posto na sua praxis, e não pelo ideal científico, na formulação do semidizer diagnóstico. Apresenta no desdobramento da escrita do caso de uma criança identificada ao diagnóstico médico e familiar que a mantinha como deficiente, uma possibilidade de discernimento que preserva a opacidade do sujeito, bem como sua articulação.

Palavras-chave: Ética. Diagnóstico. Ato. Escrita. Clínica psicanalítica. Posição do analista. 


\begin{abstract}
Maesso, M. C. (2011). Foundations of diagnosis and the position of the analyst. Doctoral Thesis, Institute of Psychology, University of São Paulo, São Paulo.

This study adopts an approach that is informed by psychoanalysis and its ethical propositions, with regard to the foundations of diagnosis. To begin with, based on the perspectives of writers such as Freud and Lacan, as well as other authors from the field of psychoanalysis, diagnosis is regarded as an act of mastery in medical discourse. It is then argued that the opposite of this discourse represents the conception of diagnosis in psychoanalysis as a "half-saying", based on the position of the analyst in the transferential relationship. The study also considers the hypothesis that through writing in clinical cases, when formulating diagnostic half-sayings, the psychoanalyst is guided by the Real encountered in their praxis, and not by the scientific ideal. By focusing on the evolution of the case writing involving a child who was diagnosed by doctors and family members as being disabled, this study advances a possible means of understanding which preserves the opacity of the subject as well as its articulation.
\end{abstract}

Keywords: Ethics, Diagnosis, Act, Writing, Psychoanalytic Clinic, Position of the analyst. 


\section{RÉSUMÉ}

Maesso, M. C. (2011). Fondements du diagnostic et la position de l'analyste. Thèse de Doctorat, Institut de Psychologie, Université de São Paulo, São Paulo.

Ce travail comprend une approche orientée par la psychanalyse et par ses propositions éthiques sur les fondements du diagnostic. Dans ce but, à travers Freud, Lacan et d'autres auteurs $d u$ domaine psychanalytique, on situe le diagnostic d'abord comme l'acte de jugement du discours médical, puis on développe l'idée que l'inverse de ce discours constitue la condition pour concevoir le diagnostic dans la psychanalyse comme le demi-dire, à partir de la position de l'analyste dans la relation transférentielle. On considère l'hypothèse selon laquelle à travers l'écriture du cas clinique le psychanalyste s'oriente grâce au Réel appliqué à sa praxis et non à l'idéal scientifique dans la formulation du demi-dire diagnostique. On présente une possibilité de discernement qui préserve l'opacité du sujet ainsi que son articulation à partir du développement de l'écriture du cas d'un enfant identifié par le diagnostic médical et familial comme handicapé.

Mots-clés: Éthique, Diagnostic, Acte, Écriture, Clinique psychanalytique, Position de l'analyste. 


\section{SUMÁRIO}

INTRODUÇÃO ..................................................................................... 11

PARTE I: ENTRE A TEORIA E A CLÍNICA ............................................ 16

1.1 Diagnosticar é um ato médico?........................................................... 16

1.2 Território inacabado da teoria........................................................... 21

1.2.1 Teorizar ou não teorizar?............................................................... 28

PARTE II: DO ALÍVIO ALIENANTE DO DIAGNÓSTICO AO TRÁGICO NA PSICANÁLISE ........................................................................ 32

2.1 Um alívio que aliena............................................................................ 32

2.2 A rede e os buracos............................................................................ $\quad 36$

2.3 Sofrimento e verdade......................................................................... 41

2.3.1 Ato de mestria................................................................................ 48

2.4. Há meios de escapar dos trilhos?......................................................... 52

PARTE III: DIAGNÓSTICO E ÉTICA DA PSICANÁLISE....................... 60

3.1 Caso terminável e interminável............................................................... 60

3.2 Diagnóstico e escrita de caso................................................................... 69

3.2.1 Escrever é preciso..............................................................................

3.2.2 O Desenhador .......................................................................... 75

CONSIDERAÇÕES FINAIS........................................................................ 96

REFERÊNCIAS...................................................................................... 98 